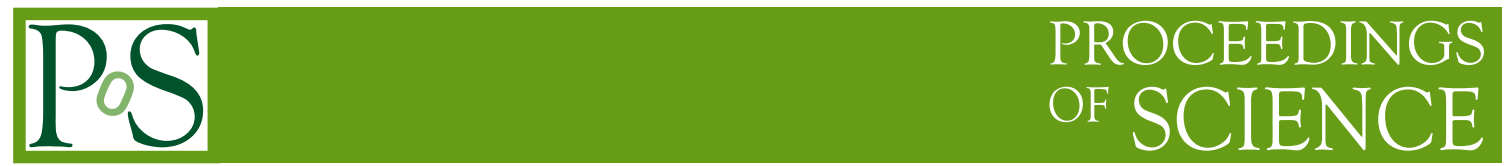

\title{
A QCD axion from a warped extra dimension
}

\author{
Thomas Flacke* \\ Rudolf Peierls Centre for Theoretical Physics \\ University of Oxford, 1 Keble Road, Oxford, OX1 3NP, UK \\ E-mail: t.flackel@physics.ox.ac.uk
}

\begin{abstract}
In this talk I am reporting on a number of realizations of axions existing in a multi- 'throat' generalization of the warped throat geometry of a Randall-Sundrum slice of $A d S_{5}$. A Peccei-Quinn scale in the phenomenologically allowed axion window is realized by warping. In compactifications with two or more throats it is shown to be possible to simultaneously solve the Standard Model hierarchy problem by the Randall-Sundrum mechanism while implementing interesting warped axion models. The constructions discussed are related to holographic duals of previously studied models of composite axions arising from strongly coupled four-dimensional dynamics.
\end{abstract}

Cargèse Summer School: Cosmology and Particle Physics Beyond the Standard Models June 30-August 112007

Cargèse, France

*Speaker. 


\section{Introduction}

The strong CP problem comprises one of the outstanding puzzles of the Standard Model (SM) of elementary particle physics. Due to the non-trivial QCD vacuum caused by instanton solutions,

$$
S_{\theta}=\frac{\bar{\theta}}{64 \pi^{2}} \varepsilon_{\mu \nu \lambda \sigma} G^{a \mu v} G^{a \lambda \sigma}
$$

is a physical $\mathrm{CP}$ violating contribution to the QCD action. In Eq. (1.1), $\bar{\theta}=\theta-\arg \operatorname{det} M_{q} \operatorname{describes}$ $\mathrm{CP}$ violation by the bare QCD vacuum $\theta$ as well as by the phase of the CKM-matrix which must be $\mathscr{O}(1)$ in order to explain the observed CP violation in the $K$ - and $B$-meson systems. However, the non-observation of a neutron electric dipole moment implies $|\bar{\theta}| \lesssim 10^{-9}$. Unless explained otherwise, this implies that the QCD vacuum contribution has to cancel the CKM-phase almost perfectly, which means severe fine-tuning.

One solution to the strong CP problem, proposed by Peccei and Quinn [1] (see also Weinberg and Wilczek [2]) is to endow the SM with additional matter content and a global $U(1)_{P Q}$ symmetry, spontaneously broken at a scale $f_{P Q}$. The associated Goldstone boson - the axion $a$ - couples to QCD via

$$
\Delta S=\frac{a}{32 \pi^{2} f_{P Q}} G \tilde{G}
$$

If the Peccei-Quinn (PQ) charges of the matter fields of the theory are assigned such that the theory has a global $U(1)_{P Q} S U(3)_{c}^{2}$ anomaly, then strong QCD dynamics generates an effective potential for $a$ such that $\bar{\theta}$ is dynamically set to zero by the vacuum expectation value of $a$. Laboratory experiments as well as astrophysical and cosmological considerations constrain the PQ scale to the "axion window" $10^{9} \mathrm{GeV} \lesssim f_{P Q} \lesssim 10^{12} \mathrm{GeV}$. Thus, solving the strong CP problem via the axion mechanism comes at a price: the hierarchy $m_{\text {weak }} \ll f_{P Q} \ll M_{\mathrm{pl}}$ must be explained by the underlying theory.

As shown by Randall and Sundrum [3], large hierarchies in energy scales can be explained naturally in models with a warped extra dimension, which commonly occurs in "throats" in string constructions with flux. Recently, several authors argued that geometries with multiple throats and a range of warp factors are not unnatural in string compactifications $[4,5,6]$. This motivates us to address the problem of the $m_{\text {weak }} \ll f_{P Q} \ll M_{\text {pl }}$ hierarchy in a multi-throat setup.

\section{An axion model on a multi-throat background}

Our model is based on a warped $5 \mathrm{~d}$ geometry with two throats - one containing the SM, in particular the Higgs and the SM fermions, while the other throat contains the axion. The geometry of both throats will be approximated by a standard Randall-Sundrum slice of $A d S_{5}$ with the metric

$$
d s^{2}=(k z)^{-2}\left(\eta_{\mu v} d x^{\mu} d x^{v}-d^{2} z\right),
$$

where for simplicity we choose a common curvature radius $1 / k$ in both throats. The $\mathrm{z}$ coordinates of the two throats lie in the ranges $z_{i} \in\left[1 / k, L_{i}\right]$, where $k L_{S M}=M_{\mathrm{pl}} / m_{\text {weak }}$ in order to establish the $M_{\mathrm{pl}}-m_{\text {weak }}$ hierarchy, and we denote $L_{P Q} \equiv L$. The positions of the UV branes are identified, i.e. the throats are glued together "back to back" (c.f. Fig. 1). In this setup, the 4D effective 


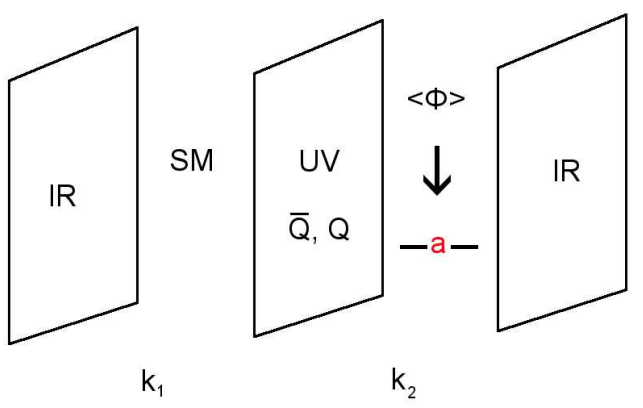

(a)

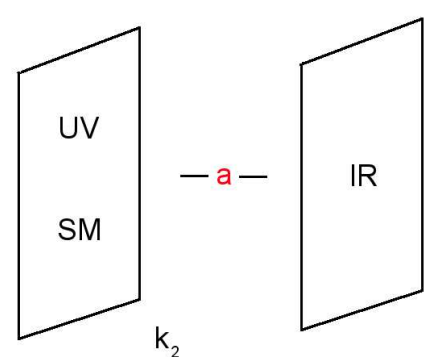

(b)

Figure 1: Illustration of a two throat axion toy model. a) The standard model lives in left $A d S_{5}$ throat while the field $\Phi$ (either scalar or gauge field) realizing the axion lives in the right throat. b) Once the PQ symmetry is broken, the axion effectively sees the Standard Model as UV confined matter.

Planck mass is given in terms of the underlying $5 \mathrm{~d}$ Planck mass $M_{5}$ by $M_{\mathrm{pl}}^{2}=2 M_{5}^{3} / k$, which only differs from the one-throat result by the factor of two.

In Ref. [7] we developed implementations of an axion from a 5D complex scalar field as well as from a 5D gauge field, which both successfully establish the desired energy scale. In both scenarios, the SM matter fields and the axion are localized in different throats, communicating only via UV boundary localized operators. Hence, we can be agnostic about the implementation in the SM throat and model all matter fields as UV brane localized operators (c.f. Fig. 1 for an illustration). We allow the gauge fields to propagate in both throats in order to be able to vary the localization of the axion-gluon coupling in the PQ throat.

In this talk I focus on the gauge field realization. When compactifying a 5D gauge field $A_{M}, A_{5}$ gives rise to a scalar degree of freedom in the 4D effective theory. If $A_{5}$ inherits a shift symmetry and couples appropriately to QCD, it satisfies all requirements to serve as an axion. As a minimal model, consider an abelian gauge field in the bulk of the PQ throat with the action

$$
S=\int d^{5} x \sqrt{-g}\left(-\frac{1}{4 g_{5}^{2}} g^{M N} g^{P Q} F_{M P} F_{N Q}-\frac{1}{2 \xi g_{5}^{2}}\left[g^{\mu v} \partial_{\mu} A_{v}+z \xi g^{55} \partial_{5}\left(\frac{A_{5}}{z}\right)\right]^{2}\right)
$$

where the gauge-fixing is chosen such that no mixing between $A_{\mu}$ and $A_{5}$ occurs. With boundary conditions

$$
\left.A_{\mu}\right|_{\mathrm{bdy}}=0,\left.\quad \partial_{5}\left(\frac{A_{5}}{z}\right)\right|_{\mathrm{bdy}}=0
$$

the $A_{\mu}$ zero-mode is projected out, while the $A_{5}$ zero-mode is IR localized

$$
A_{5}(x, z)=N z a(x),
$$

where $N \approx \sqrt{2 k g_{5}^{2}} / L$ is determined from the canonical normalization of the kinetic term for $a(x)$. The gauge transformations which leave the boundary conditions Eq. (2.3) invariant, correspond to a global shift symmetry on $a(x)$ [7]. The axion-gluon coupling can be achieved via a mixed ChernSimons term. In the simplest setup with all SM fields (including the gluon) in the SM throat, the 
coupling must be localized on the UV brane where - due to the imposed boundary conditions - only $A_{5}$ and the $4 \mathrm{D}$ gluon zero-mode are non-vanishing. The mixed Chern-Simons term is

$$
S_{\text {int }}=\left.\int d^{4} x \frac{\alpha}{64 \pi^{2} M_{5}} A_{5}\right|_{\mathrm{UV}} \varepsilon^{\mu \nu \lambda \sigma} G_{\mu \nu}^{a} G_{\lambda \sigma}^{a}
$$

where $G$ denotes the QCD field strength and $\alpha$ is an $\mathscr{O}(1)$ constant. This interaction precisely corresponds to the axion-gluon coupling in Eq. (1.2). However in this 5D model, the Peccei-Quinn scale is calculable. Canonical normalization of the kinetic term for $a(x)$ yields $f_{P Q}=M_{5} k L / \sqrt{2 g_{5}^{2}}$. For natural parameters (with the standard assumptions $M_{\mathrm{pl}} \gtrsim M_{5} \gtrsim k$ and $g_{5}^{2} \sim 1 / M_{5}$ ) this implies $f_{P Q} \sim M_{5}^{2} L \gg M_{5} \sim M_{\mathrm{pl}}$, i.e. $f_{P Q}$ is warped up due to the volume suppression of the coupling, rather than warped down as desired.

The situation improves if QCD is assumed to propagate in both throats. Now the 5D ChernSimons term appears in the whole PQ throat:

$$
S_{i n t}=\int d^{5} x \frac{\alpha}{64 \pi^{2} M_{5}} \varepsilon^{S M N P Q_{A} G_{M N}^{a} G_{P Q}^{a}}
$$

As the $A_{\mu}$ zero-mode is projected out, Eq. (2.6) yields the interaction

$$
S_{\text {int }}=\int d^{5} x \frac{\alpha}{64 \pi^{2} M_{5}} A_{5} \varepsilon^{\mu \nu \lambda \sigma} G_{\mu \nu}^{a} G_{\lambda \sigma}^{a}
$$

in terms of the light fields of the effective theory. Here, the gluon kinetic term must be normalized as well, and the axion coupling is not volume suppressed. This scenario leads to

$$
f_{P Q}=\sqrt{\frac{2}{k g_{5}^{2}}} \frac{1}{L_{1}} \simeq \frac{M_{\mathrm{pl}}}{M_{5} L_{1}} .
$$

$f_{P Q}$ is warped down to the IR brane scale $1 / L_{1}$. To obtain a phenomenologically viable PQ scale we require a PQ throat with warping $k L=10^{6} \ldots 10^{9}$, which is milder than the standard warping required for the SM throat $\left(k L_{S M}=M_{\mathrm{pl}} / m_{\text {weak }} \sim 10^{15}\right)$. The mass of the first Kaluza-Klein excitation of $A_{\mu}$ is of order $1 / L_{1} \sim f_{P Q}$, not imposing any further phenomenological constraint. We conclude that the setup provides a phenomenologically viable, natural realization of the $m_{\text {weak }} \ll f_{P Q} \ll M_{\mathrm{pl}}$ hierarchy.

Apart from successfully creating the hierarchies, the realization of the axion via a $5 \mathrm{D}$ gauge field on $A d S_{5}$ provides two further advantages:

- Protection of the axion potential by a gauge symmetry:

A common problem for axion models arises because the axion potential is very sensitive to corrections which can shift the axion vev. The potential is protected by the global U(1) symmetry, but global symmetries are generically expected to be broken by quantum gravity effects such that higher dimensional Planck suppressed operators must be forbidden by other means [8].

In the model presented here, higher dimensional operators are forbidden by a U(1) gauge symmetry which - due to the no-hair theorem - even holds when gravity becomes strong. 
- Dual interpretation as a composite axion model:

Using the AdS/CFT correspondence, ${ }^{1}$ the axion realization presented here is dual to composite axion model as summarized in Table 1 and discussed in detail in Ref. [7]. Composite axion models, in which $f_{P Q}$ is explained by dimensional transmutation, have been proposed a long time ago, but calculational power has been limited because of strong coupling. We presented a dual $A d S_{5}$ model which is weakly coupled and perfectly calculable.

\begin{tabular}{|c|c|c|}
\hline$A d S_{5}$ & $\leftrightarrow$ & 4D $C F T$ \\
\hline 5D $U(1)$ gauge symmetry & $\leftrightarrow$ & global $U(1)$ symmetry \\
\hline IR Dirichlet b.c. on $A_{\mu}$ & $\leftrightarrow$ & global $U(1)$ is spont. broken \\
\hline$\rightarrow$ IR Neumann b.c. on $A_{5}$ & $\leftrightarrow$ & Goldstone boson $(a)$ \\
\hline$A_{5}$ is IR localized & $\leftrightarrow$ & $a$ is composite \\
\hline UV Dirichlet b.c. on $A_{\mu}$ & $\leftrightarrow$ & $U(1)$ is explicitly broken \\
\hline$\rightarrow$ UV Neumann b.c. on $A_{5}$ & $\leftrightarrow$ & $a$ acquires a (small) mass \\
\hline
\end{tabular}

Table 1: $A d S / C F T$ correspondence of the $5 \mathrm{D}$ axion from $A_{5}^{(0)}$ with a composite $4 \mathrm{D}$ axion.

\section{Acknowledgments}

I would like to thank the organizers, lecturers and participents of the Cargèse Summer School 2007 on Cosmology and Particle Physics Beyond the Standard Models for providing a very stimulating atmosphere for this school, for their shared insight and many interesting discussions. I am furthermore grateful to the Oxford Physics Department for financial support.

The work presented in this talk was supported by "Evangelisches Studienwerk Villigst e.V.", PPARC Grant No. PPA/S/S/2002/03540A, and the EC 6th Framework Programme MRTN-CT-2004-503369.

\section{References}

[1] R. Peccei and H. Quinn, Phys. Rev. Lett. 38, 1440 (1977); Phys. Rev. D16, 1791 (1977).

[2] S. Weinberg, Phys. Rev. Lett. 40, 223 (1978); F. Wilczek, Phys. Rev. Lett. 40, 279 (1978).

[3] L. Randall and R. Sundrum, Phys. Rev. Lett. 83, 3370 (1999)

[4] S. Dimopoulos, S. Kachru, N. Kaloper, A. E. Lawrence and E. Silverstein, Phys. Rev. D 64, 121702 (2001); Int. J. Mod. Phys. A 19, 2657 (2004).

[5] G. Cacciapaglia, C. Csaki, C. Grojean and J. Terning, Phys. Rev. D 74, 045019 (2006).

[6] A. Hebecker and J. March-Russell, arXiv:hep-th/0607120.

[7] T. Flacke, B. Gripaios, J. March-Russell and D. Maybury, JHEP 0701 (2007) 061.

[8] M. Kamionkowski and J. March-Russell, Phys. Lett. B 282 (1992) 137.

[9] R. Contino, Y. Nomura and A. Pomarol, Nucl. Phys. B 671 (2003) 148.

\footnotetext{
${ }^{1}$ See Ref. [9] for the dual interpretation of gauge fields on $A d S_{5}$.
} 